Article

\title{
Miniaturized Dielectric Disc Loaded Monopole Antenna
}

\author{
Khan Masood Parvez ${ }^{1 *}$, SK. Moinul Haque ${ }^{1}$ and Laxmikant Minz ${ }^{2}$ \\ 1 Department of Electronics \& Communication Engineering, Aliah University, Newtown, Kolkata, India, \\ masoodpavez1993@gmail.com \\ 2 Korea Advanced Institute of Science and Technology 5207, E3-2, 291 Daehak-Ro, Yuseong-gu, Dae- \\ jeon, 34141, South Korea, laxmikant@syratron.com \\ * Correspondence: masoodparvez1993@gmail.com; Tel.: +91 7699559488
}

\begin{abstract}
This paper deals with miniaturization technique based on frequency reduction using top loaded dielectric discs. In contrast to a simple monopole, the resonant frequency of monopole loaded with two dielectric discs changes from 1.98 to $1.29 \mathrm{GHz}$, resulting $34.84 \%$ reduction in resonant frequency keeping the antenna length $(36.00 \mathrm{~mm})$ unaltered. It is well-known fact that dielectric material can trap the energy to be delivered from source to antenna and as a result, it is unable to radiate efficiently. Then any approach to use the dielectric material for miniaturization process must, therefore, antenna coupled in such a way that it can radiate efficiently. The dielectric disc on top of monopole creates an inductive situation in a similar way to oppositely directed wire loop compensate the capacitive effect present at monopole causes the reduction in resonant frequency. This concept is implemented without sacrificing any desired features like bandwidth, radiation characteristics and efficiency (more than 98\%) and analyzed with an equivalent circuit model. Experimental results illustrate good agreement with simulation results. This monopole antenna in car can be designed for GPS system, car to car communication, GSM or CDMA operation.
\end{abstract}

Keywords: Dielectric disc loaded, miniaturization, monopole antenna

\section{Introduction}

The monopoles are the simplest and most widely used class of antennas for almost a century since the innovation of wireless radio communication. These antennas are fed by the coaxial connector with its central conductor connected through a hole in the ground plane to the vertical monopole structure and its outer conductor connects to the ground plane. When we introduce the image, the monopole is virtually identical to a dipole for analysis. The fields disappear below the ground plane and restricting the fields to the upper hemisphere doubles the gain over a dipole since only half of the input power of the dipole is needed to generate the same field strength. It is omnidirectional radiation profile. The quarter wave antennas are also appropriate for the network for unattended ground sensors, mobile communication and terrestrial applications and situation where earth surface can be used as an infinite ground plane. Therefore, investigations of electrically small monopoles are given the opportunity to fulfill the demand for compact wireless systems. The small antennas are related to interchange of the electrical and physical properties of an antenna. The monopole antenna has " $k a$ " value less than or equal to 0.5 is known to be an electrically small antenna [1]-[2], where " $k$ " is the wave number in free space and " $a$ " is the radius of the smallest sphere circumscribing the antenna. In [3], "ka" value for single loop loaded 
monopole is 0.58 which is very close to the limit. The dipole current creates the capacitive store energy by its electric field in near field region is compensated by an induced magnetic field offered by the loop. The monopole is also loaded by similar loop and reported $23.88 \%$ reduction in resonant frequency with $96.10 \%$ efficiency. An approach for electrically small monopole antenna in [4] is based on multi elements. In reference [5], the peano-curve loaded monopole is reported to operate at $2.45 \mathrm{GHz}$ frequency. A miniature dielectric loaded monopole antenna for $2.4 / 5 \mathrm{GHz}$ WLAN applications is presented in [6]. Top loaded monopole with shorting post is studied in [7]. Slot antenna miniaturization techniques have been delineated in [8]-[10]. Antenna miniaturization using high permittivity material has been reported in [11]-[12]. The sinusoidal-Galerkin moment method for monopole antenna at centre of circular disc has been described in [13]. Wheeler's design criteria for electrically small antenna designs with theoretical formulations have been briefly illustrated in [14]. The disc monopoles have been addressed for input impedance matching [15] and time domain analysis [16]. The monopole antenna horizontally loaded with circular disc have been presented for enhance bandwidth in literature [17]-[20]. Printed circular, elliptical, square, rectangular, and hexagonal disc monopole antennas have been highlighted for wide band applications in [21].

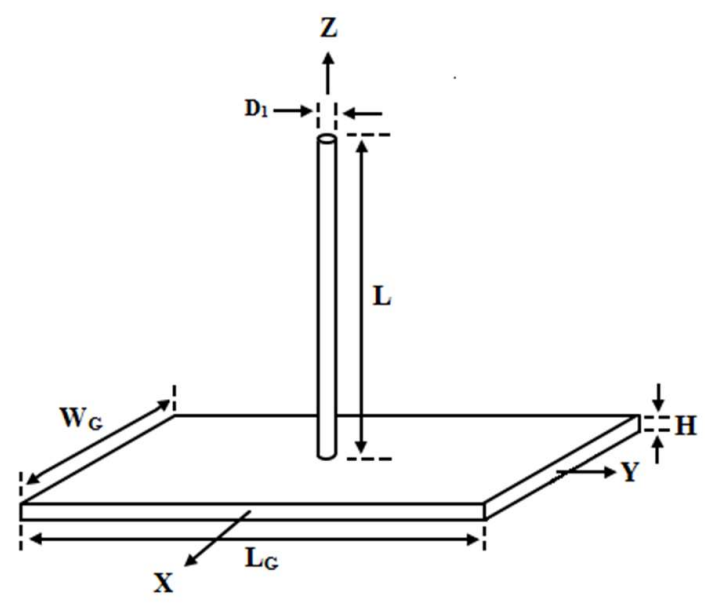

Figure 1. Monopole

Although top loaded disc is well established concept for bandwidth enhancement and mathematical analysis, it effects on miniaturization has not been extensively studied. In this current approach, the length of quarter wave monopole and proposed antenna are same. Hence, without increasing the monopole length, we try to reduce the resonant frequency in higher degree comparison with existing literature. So, It can adversely hamper the antenna performances at particular when we try to reduce the resonant frequency with unaltered antenna length in return loss, radiation pattern, input impedance, efficiency etc. It is always a challenging task for research community to develop the miniaturized antenna with less adverse effect on antenna performances. 


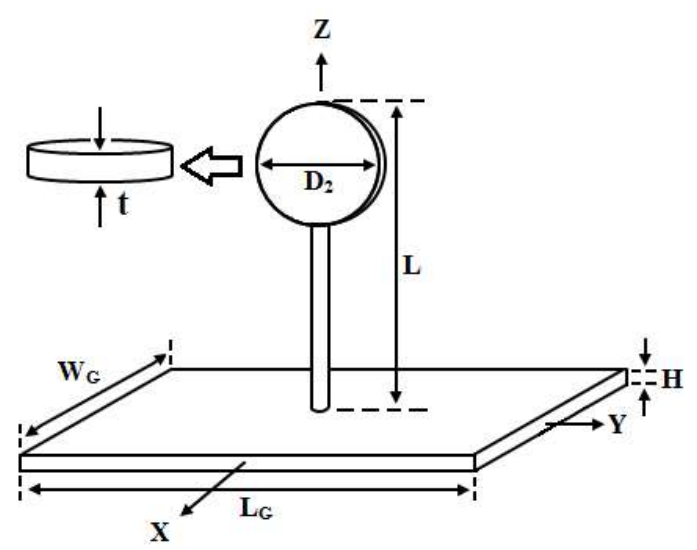

Figure 2. Monopole antenna loaded with a single dielectric disc.

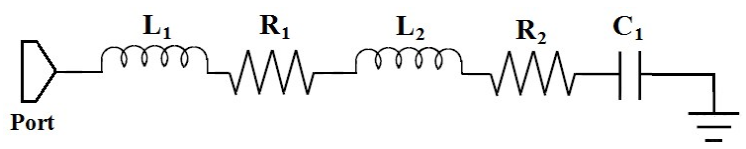

Figure 3. Equivalent circuit model for proposed monopole antenna loaded with single dielectric disc.

In this study, a small monopole antenna is presented based on frequency reduction technique using top loaded dielectric discs. Firstly, the simple monopole is efficiently coupled with one dielectric disc on top of monopole in similar way coupled to opposite directed wire loop [8]. This concept yields 25.75\% reduction on resonance frequency keeping monopole length unaltered to bring it much close to electrically small limit. In order to achieve more reduction in resonant frequency, we have introduced one more circular disc on the top of monopole. And as a result, the proposed antenna produces $34.84 \%$ reduced resonant frequency with $22.55 \%$ bandwidth and $97.18 \%$ efficiency. An equivalent circuit model is also proposed to better understand the inductive effect on monopole. The " $k a$ "[1]-[2] value for monopole antenna loaded with single and double dielectric discs are 0.55 and 0.48 respectively without imposing any additional matching network.

\section{Single dielectric disc loaded monopole antenna miniaturization}

\subsection{Description of antenna structure}

It is well known that the length of monopole is inversely proportional to antenna resonance frequency. Any approach to reduce the resonant frequency with unaltered antenna length is a crucial task for the researcher community due to its adverse effect on radiation pattern, gain, bandwidth, efficiency etc. The schematic diagram of monopole antenna is shown in figure 1. This antenna is considered as a reference antenna to better comprehend the reduction of resonant frequency keeping antenna length unchanged. The length $(L)$ and diameter $(D)$ of monopole structure are $36.00 \mathrm{~mm}$ and $1.00 \mathrm{~mm}$ respectively. 


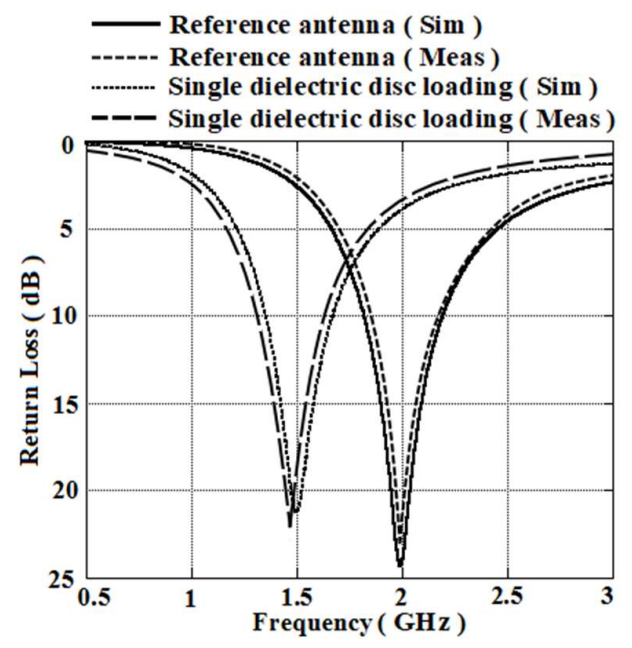

Figure 4. Equivalent circuit model for proposed monopole antenna loaded with single dielectric disc.

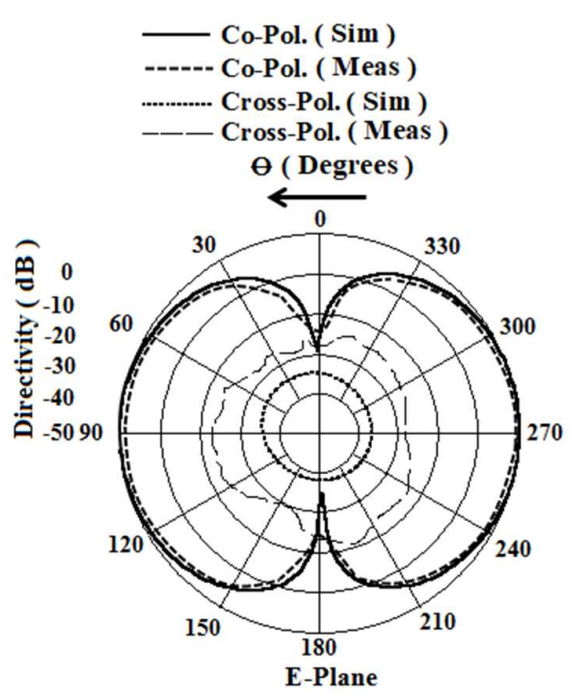

(a)

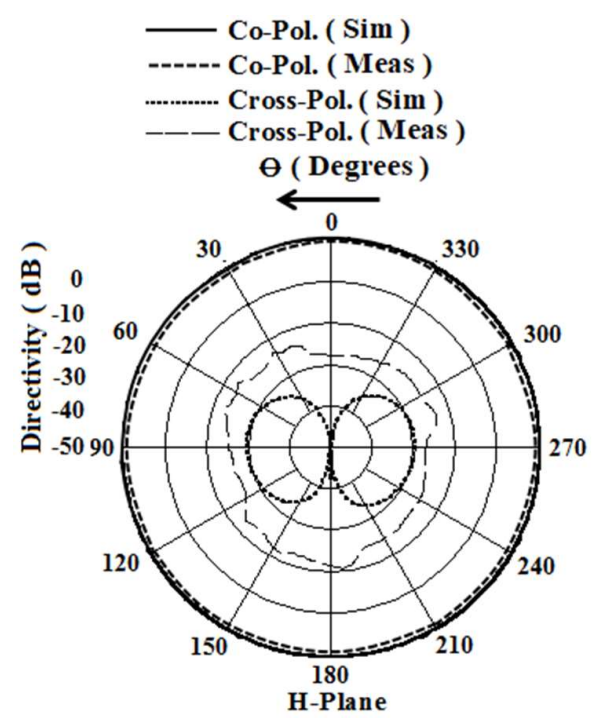

(b)

Figure 5. Radiation characteristics of single dielectric disc loaed monopole antenna at resonance frequency (a) E-Plane (b) H-Plane.

The rectangular copper ground plane is used to construct the antenna with the dimension of $50.00 \mathrm{~mm} \times 50.00 \mathrm{~mm} \times 0.90 \mathrm{~mm}\left(L_{G} \times W_{G} \times H\right)$ considering its finite conductivity of $5.8 \times 10^{7}$ Siemens $/ \mathrm{m}$. The current on monopole structure excites to induce the electric field along the monopole in the near field region. The capacitive reactive environment below the resonant frequency of monopole antenna leads to enhance stored energy which is responsible for high quality factor ( $Q$ ). One possible methodology [3] to cancel out the capacitive effect is to introduce inductive environment on monopole structure such way that it is well coupled with the monopole structure. 
The inductive effect can be utilised using metallic loop. Here, reactance of monopole and loop are oppositely directed. This strategy had been used to miniaturize dipole, monopole and slot antenna in [3]. Here we replaced the conductive loop to circular dielectric disc. Use of dielectric not only provides us higher degree of miniaturization but also better efficiency having not use metal loop. It is well known fact that antenna configuration with high dielectric material [11] can trap the delivered energy to the antenna for its high dielectric constant. Any attempt to construct a miniaturized antenna using dielectric material must, therefore have to be coupled in such a manner that it can radiate efficiently. We have used RT/Duriod 6010 LM substrate ( $\varepsilon=10.2, \delta=0.0023$ ) to implement the present the concept. The proposed antenna does not require any additional matching network.

Figure 2 illustrates the single disc loaded monopole antenna. So, the proposed antenna itself behaves as a self-resonant structure [2]-[3]. The diameter ( $\left.D_{2}\right)$ and thickness ( $t$ ) of dielectric substrate are $21.00 \mathrm{~mm}$ and $2.54 \mathrm{~mm}$ respectively. The monopole length ( $L$ ), diameter $\left(D_{1}\right)$ and ground plane size $\left(L_{G} \times W_{G} \times H\right)$ are same as reference monopole of figure 1 . The antenna simulations were carried out using the full-wave electromagnetic solver Ansys HFSS v.19.2 [22].

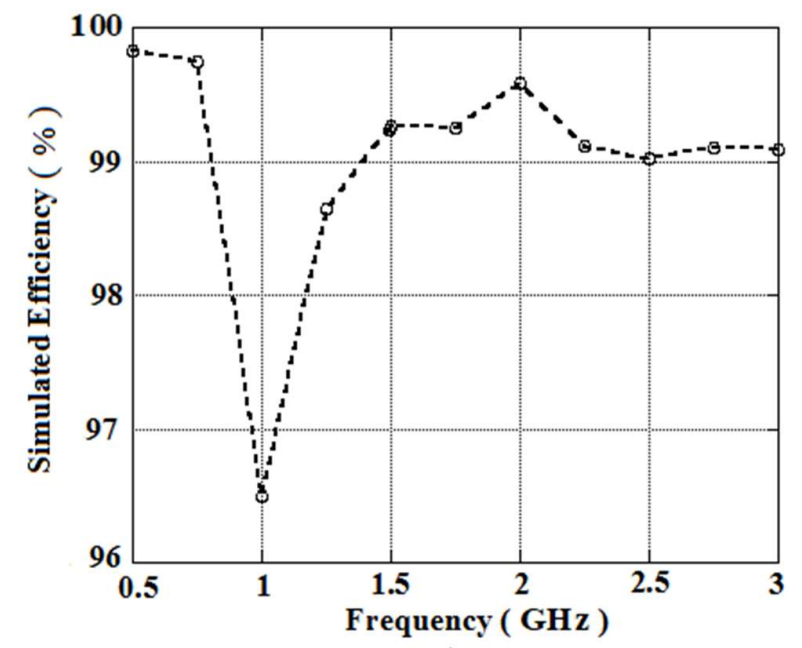

Figure 6. Simulated efficiency verses frequency plot for single dielectric loaded monopole antenna.

\subsection{Equivalent circuit model}

The equivalent circuit model of single dielectric disc loaded monopole antenna has been highlighted in figure 3.The circuit model has been analysed using NI AWR [23] software. The parametric values are as follows: $\mathrm{L} 1=10.87 \mathrm{nH}, \mathrm{L} 2=0.2683 \mathrm{nH}, \mathrm{R} 1=42.1 \Omega$, $\mathrm{R} 2=1 \times 10-8 \Omega, \mathrm{C} 1=1.001 \mathrm{pF}$. Here all symbols have their usual meaning.

\subsection{Results and description}

Figure 4 presents the return loss plot for unloaded monopole antenna and disc loaded monopole. The simulated results are compared with measured results and found agreeing well each other. The operating frequency for proposed antenna is shifted towards the left side in return loss in comparison with the unloaded monopole. The antenna length ( $L$ ) of unload monopole and loaded monopole antenna are same. Hence reduction in resonant frequency is achieved without changing the antenna length of monopole. The simulated value for unload monopole is $1.99 \mathrm{GHz}$. The simulated resonant frequency for disc loaded monopole is $1.49 \mathrm{GHz}$. The measured resonance frequency for ordinary monopole antenna is $1.98 \mathrm{GHz}$ and disc loaded is $1.47 \mathrm{GHz}$, producing $25.75 \%$ reduction in resonance frequency in comparison with ordinary monopole. It is also noticeable that dielectric disc 
in monopole did not trap the delivered energy to the antenna for its high permittivity. The bandwidth $(-10 \mathrm{~dB})$ for top loaded dielectric disc is $20.66 \%$ and that value for reference monopole antenna is $14.73 \%$. Antenna is the largest component of all wireless communication systems. However, as wireless communication systems continue to evolve, the large size of the antenna is problematic. Therefore, it is imperative to give emphasis on simple and easy to implement miniaturization techniques. Proposed antennas are very simple and easy to implement comparison with miniaturized top hat loaded, complementary split ring resonator ( CSRR ) based antenna [24] and inductively coupled capacitive loaded miniaturized monopole [25].

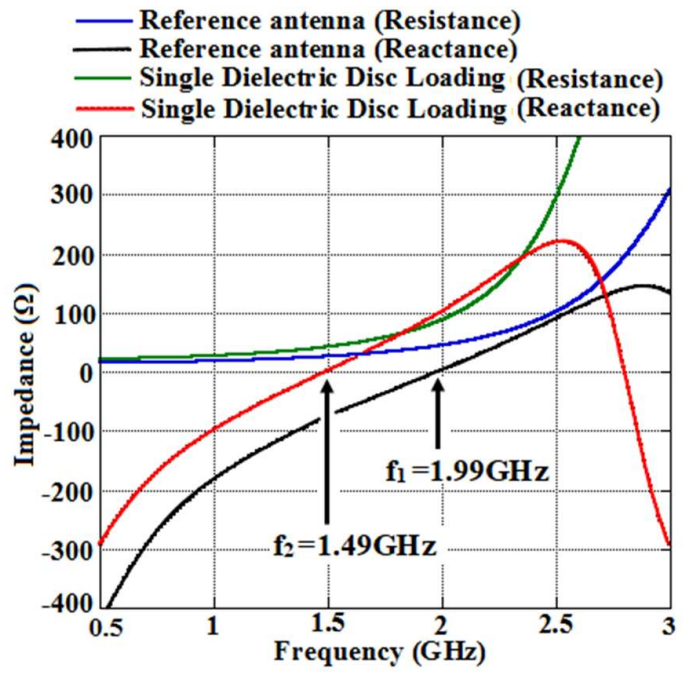

Figure 7. Simulated input impedance plot of reference monopole antenna and single dielectric loaded monopole antenna.

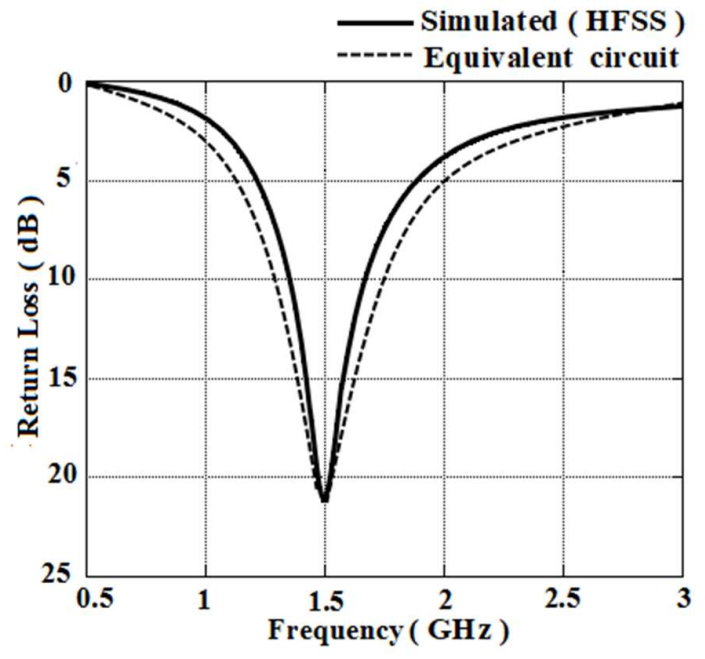

( a ) Frequency response.

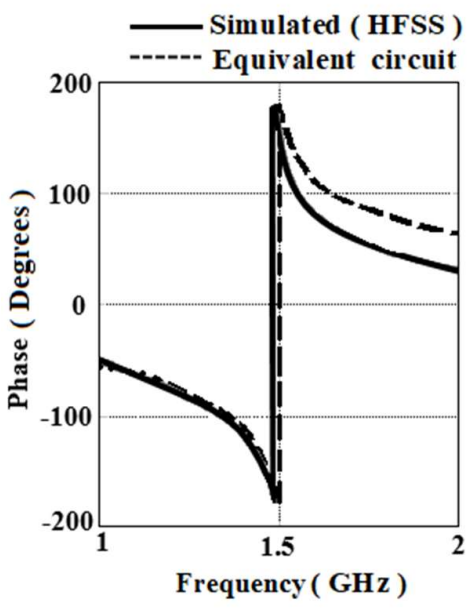

(b) Phase response

Figure 8. Simulated and equivalent circuit response of monopole loaded with single dielectric disc. ( a ) Frequency response. ( b ) Phase response

The normalized radiation characteristics of proposed monopole are illustrated in figure 5 (a) and (b). The cross-pol level of both $\mathrm{E}$ and $\mathrm{H}$ plane are below the accepted value.The dielectric disc in monopole antenna has integrated in such a way that it can radiate efficiently in distributed and symmetric manner. Hence the single dielectric disc does not distort the radiation characteristics unlike [24] relative to reference. 
The Wheeler Cap method [26] is used to measure efficiency. The efficiency can be found as $\eta=\left(R_{1}-R_{2}\right) / R_{1}$ where $R_{1}$ is the real part of the measured input impedance without the metallic cap and $R_{2}$ the real part of the measure input impedance with the metallic cap where we have enclosed the antenna. The measured efficiency and gain are $97.41 \%$ and $-1.26 \mathrm{dBi}$ respectively.The efficiency plot has been shown in figure 6 where values varies from 99 to $100 \%$ except in frequency range of 0.80 to $1.38 \mathrm{GHz}$.

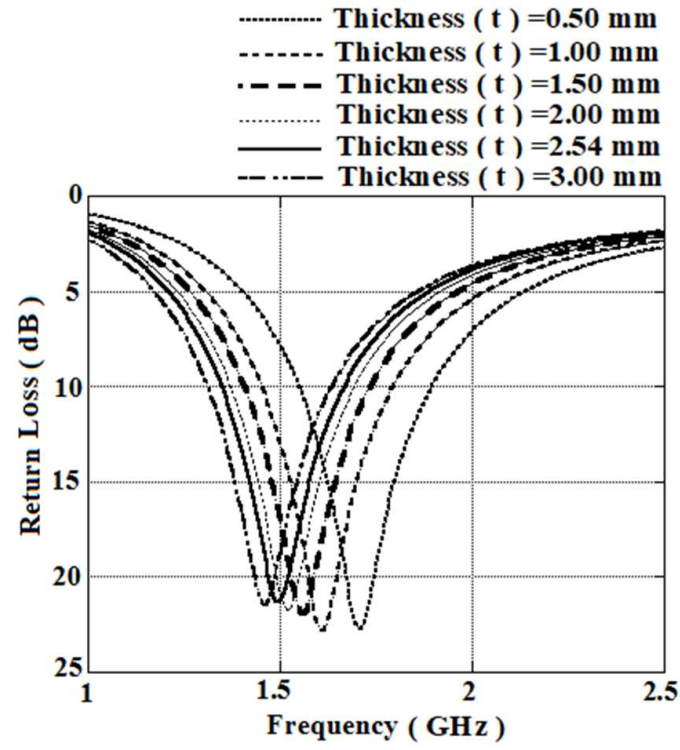

Figure 9. Simulated return loss of single dielectric loaded monopole with change in dielectric thickness.

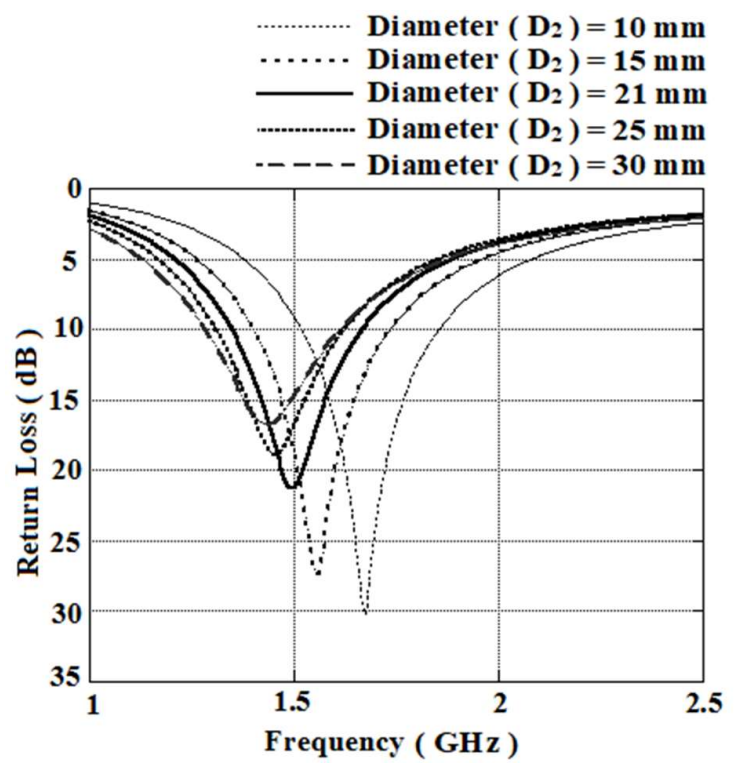

Figure 10. Simulated return loss of single dielectric loaded monopole with change in dielectric diameter. 


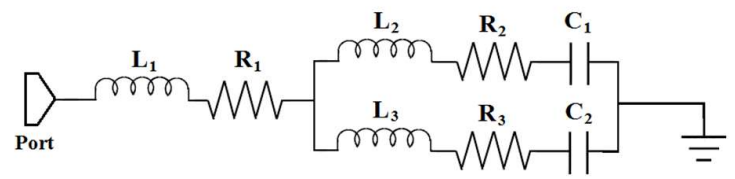

Figure 11. Equivalent circuit model for proposed monopole antenna loaded with double discs.

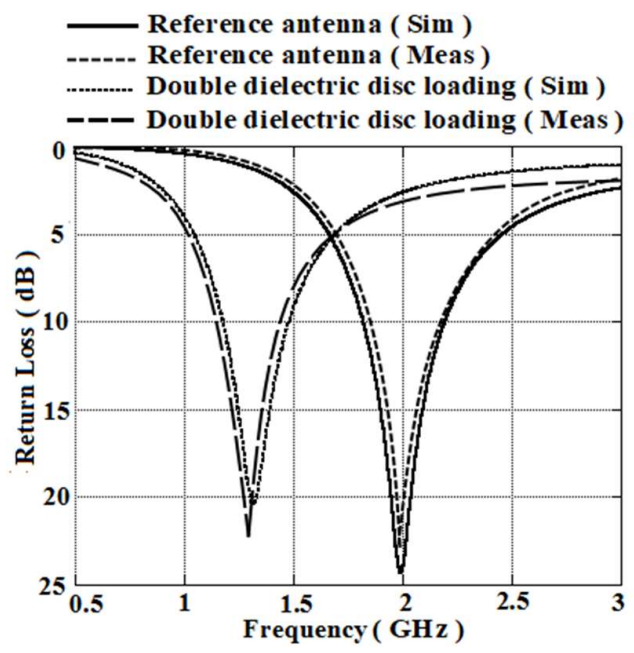

Figure 12. Return loss of reference antenna and monopole antenna loaded with double dielectric disc.

The input impedance for the proposed configuration has been illustrated in figure 7 . It is seen that the reactance for reference monopole at frequency of $1.99 \mathrm{GHz}$ touches the horizontal axis and its corresponding input resistance is $50 \Omega$. After introduction of single dielectric disc in monopole, the reactance presents at $1.49 \mathrm{GHz}$ of reference monopole antenna has been cancelled out by the inductive reactance of circular dielectric disc. This results in reduction of resonant frequency of $25.12 \%$ (simulation value) unlike in [27] where miniaturization is achieve using very high refractive index meta-material. It can be noted that the proposed antenna has match the required impedance characteristic without having any matching network.

In [28], a RLC resonant circuit and RL circuit had been used to model circular loop and feed line respectively. In current approach, an equivalent circuit has been designed combining these two circuits which is shown in figure 3 . The resonant frequency is $f=1$ / $[2 \pi \sqrt{ }(\mathrm{LC})]$. The equivalent circuit has been analysed by using NI AWR [23] simulation tool circuit and validate with antenna simulation. The values of circuit component are listed along with circuit model in figure 3 . High conductivity materials have very low resistance. For $\mathrm{R}_{2}$ resistance, we can use copper, silver, gold etc. Frequency and phase response for both the equivalent circuit and single dielectric disc loaded monopole antenna have been shown in figure 8 (a) and (b) respectively. It is seen that the simulated resonance frequency from HFSS is almost exactly followed by the circuit frequency. Excellent agreement is also fund in phase response too.

\subsection{Parametric variation}

The effect of change in disc thickness $(t)$ on return loss is shown in figure 9. For $(t)=$ $0.50 \mathrm{~mm}$, the proposed topology resonates at $1.70 \mathrm{GHz}$, resulting $14.57 \%$ reduction in resonant frequency. But interestingly, when we have increased disc thickness from $0.50 \mathrm{~mm}$ to $3.00 \mathrm{~mm}$, the return loss plot gradually move from 1.70 to $1.45 \mathrm{GHz}$ respectively. It is observed form the figure 9 that the monopole with $2.54 \mathrm{~mm}$ thickness produces $1.49 \mathrm{GHz}$ 
.Beyond the value of $3.00 \mathrm{~mm}$, the the resonant frequency does not decreases anymore. In this case, we have chosen the standard thickness ( $2.54 \mathrm{~mm}$ ) of RT Duroid 6010LM dielectric materiel for fabrication and measurement purposes.The results of variation in diameter $\left(D_{2}\right)$ of the dielectric disc are shown in figure10. It is seen that the resonant frequency is shifting towards the left side in the return loss with increment of the dielectric disc diameter $\left(D_{2}\right)$. For disc diameter of $10.00 \mathrm{~mm}$, the resonant frequency is $1.67 \mathrm{GHz}$. When diameter $\left(D_{2}\right)$ of dielectric disc is increased from $21.00 \mathrm{~mm}$ to $30.00 \mathrm{~mm}$, the return loss characteristics marginally shifted towards the left sides with poor matching in return loss. The length $(L)$ of loaded monopole antenna is $36.00 \mathrm{~mm}$. Then large disc is not also suitable for mechanical stability.

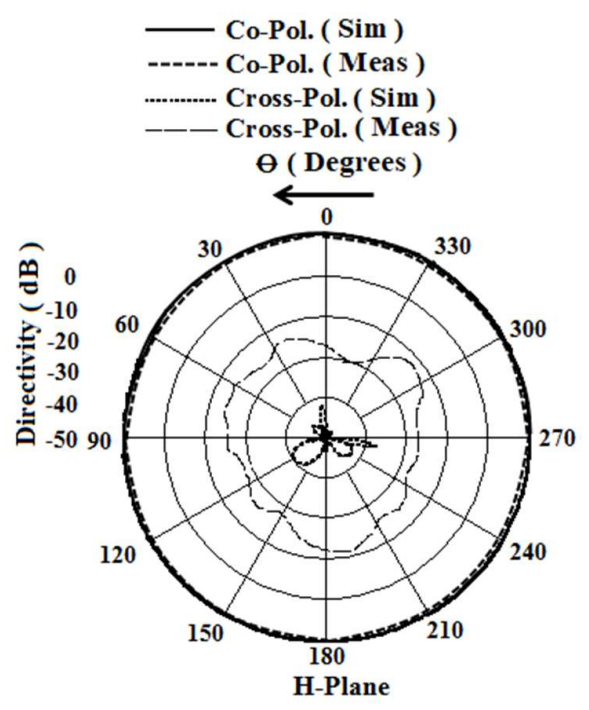

(a)

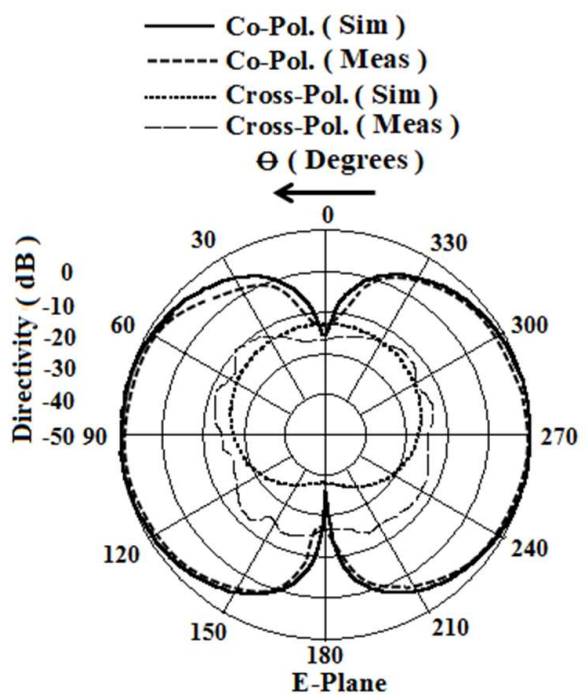

(b)

Figure 13. Radiation characteristics of monopole antenna loaded with double dielectric discs at resonance frequency (a) E-Plane (b) H-Plane.

\section{| Electrically small limit}

The " $k a$ " value for this proposed antenna is 0.55 which is very close to the electrically small antenna limit for the grounded structure. The " $k a$ "value of an antenna is defined in [2] as $k a=\left(2 \pi / \lambda_{0}\right) \times$ a where $\boldsymbol{\lambda}_{0}$ is the wavelength of the proposed antenna at resonance frequency of $1.47 \mathrm{GHz}$ and " $a$ " is the radius of the smallest sphere circumscribing the antenna. We have calculated the " $k a$ " value in similar way used in [3].

\section{Double dielectric discs loaded monopole antenna miniaturization}

\subsection{Description of antenna structure}

The dimensions of double dielectric disc loaded monopole antenna are same as described in figure 2, except here we have used two dielectric discs parallel to each other on top of monopole antenna. The fabricated double dielectric discs antenna is shown in figure 17.

\section{2 | Equivalent circuit model}


The equivalent circuit model analysis of double dielectric discs loaded monopole has been addressed in figure 11. The value for the parameters are as follows: L1 = 7.09 $\mathrm{nH}, \mathrm{L} 2=111.5 \mathrm{nH}, \mathrm{L} 3=8.862 \mathrm{nH}, \mathrm{R} 1=1 \times 10-5 \Omega, \mathrm{R} 2=165.1 \Omega, \mathrm{R} 3=42.45 \Omega, \mathrm{C} 1=0.023 \mathrm{pF}$, $\mathrm{C} 2=0.9278 \mathrm{pF}$ and for conductive materials L1 $=0.7855 \mathrm{nH}, \mathrm{L} 2=565.1 \mathrm{nH}, \mathrm{L} 3=11.01 \mathrm{nH}$, $\mathrm{R} 1=1 \times 10-11 \Omega, \mathrm{R} 2=0.1 \Omega, \mathrm{R} 3=39.22 \Omega, \mathrm{C} 1=1 \times 10-9 \mathrm{pF}, \mathrm{C} 2=1.482 \mathrm{pF}$. The symbols have their usual meaning.

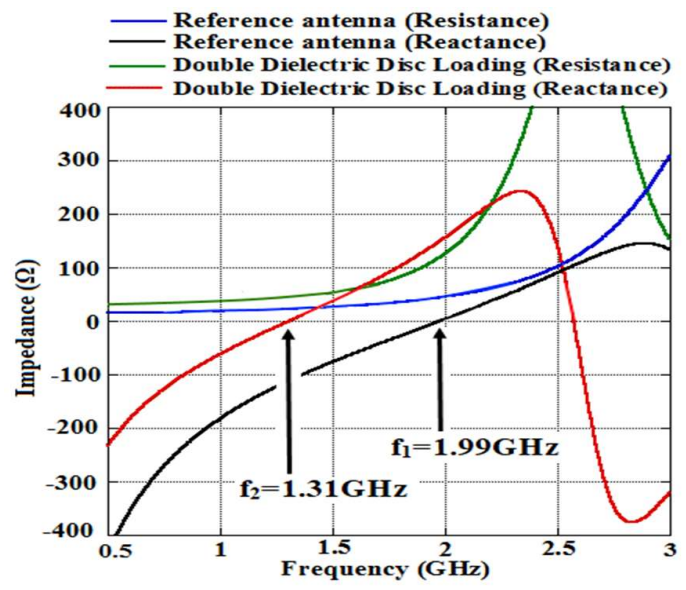

Figure 14. Simulated input impedance plot of reference monopole antenna and double dielectric loaded monopole antenna

\subsection{Results and description}

The simulated resonance frequency for double dielectric disc attached monopole antenna is $1.31 \mathrm{GHz}$ at $-20.23 \mathrm{~dB}$. The measured resonant frequency is $1.29 \mathrm{GHz}$. The reduction of resonant frequency is $34.84 \%$. The excellent agreement has been found between simulated and measured results shown in figure 12 . The $-10 \mathrm{~dB}$ impedance bandwidth is $22.52 \%$. The proposed antenna is itself an electrically small antenna due to its " $k a$ " value 0.48 at resonance frequency $1.29 \mathrm{GHz}$ without any external matching network.

The simulated and measured radiation characteristics of double dielectric discs loaded monopole have shown in figure $13(\mathrm{a})$ and $(\mathrm{b})$ respectively. The cross-pol levels in both the cases are well below $-20 \mathrm{~dB}$. Thus, introduction of double dielectric discs does not having any degrading influence on radiation characteristics. The measured efficiency for this proposed antenna is $97.18 \%$. The measured gain is $-1.58 \mathrm{dBi}$.

The impedance plot of reference and double dielectric discs loaded are shown in figure 14. It can easily be seen that input resistance for double dielectric discs is $50 \Omega$ and its associated reactance value is zero at resonant frequency of $1.31 \mathrm{GHz}$.

The equivalent circuit has been shown in figure 11. Simulation results from HFSS software and equivalent circuit results from NI AWR software of frequency and phase response are shown in figure 15 (a) and (b).

Figure 16 illustrates the current distribution on dielectric on top surface. It was seen that the proper coupling between loop and monopole or dipole creates an effective and efficient approach for miniaturization [3]. It is noticeable that direction of current flows of reference monopole and loaded monopole are opposite in nature as shown in figure 16. 
The parametric variations are also similar to single dielectric disc loaded monopole and not repeated here for brevity.
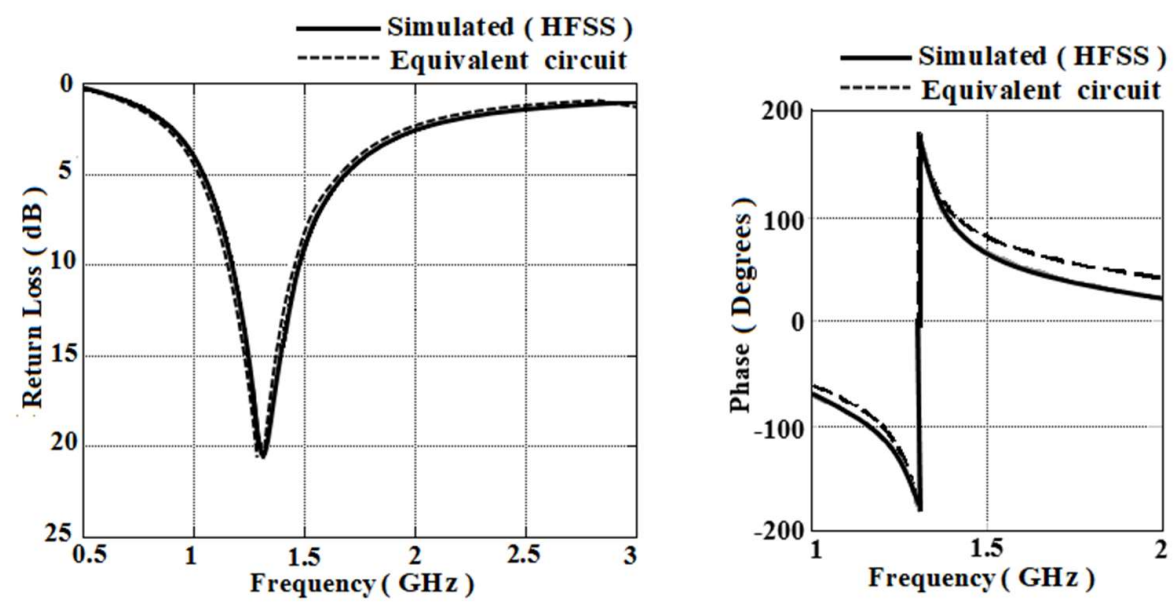

Figure 15. Simulated and equivalent circuit response of monopole loaded with double dielectric discs. ( a ) Frequency response ( b ) Phase response

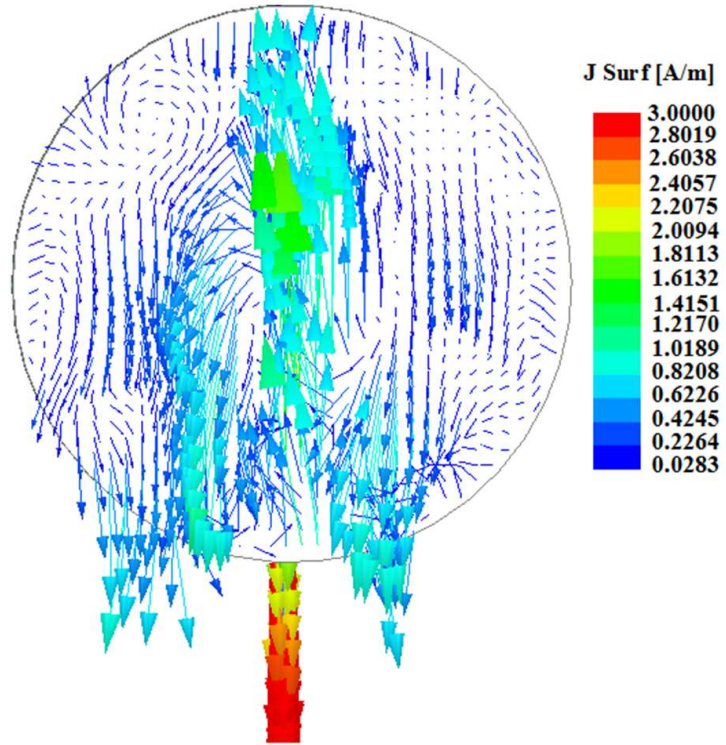

Figure 16. Current distributions on double dielectric discs

Though different network matching approaches has been noted in [29], but in our design we have not used any additional matching network. The two drawbacks -low efficiency and low input impedance in [30] for antenna miniaturization using high permittivity material have been overcome by this proposed antenna topology. Any strategies to reduce the resonant frequency of an antenna keeping the length unchanged can affect the antenna performances. It is clearly shows from the simulated and measured results that proposed miniaturization technique is introduced without sacrificing any desired features like return loss, input impedance, bandwidth, radiation characteristics and efficiency. 


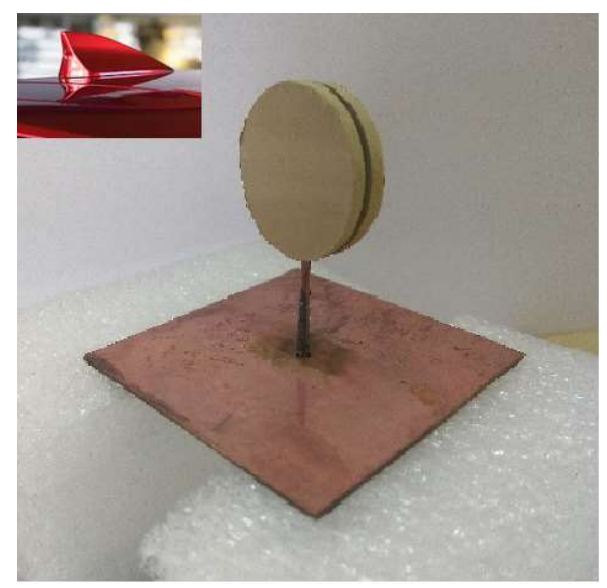

Figure 17. Fabricated prototype of monopole antenna loaded with double dielectric discs and shark-fin antenna on top left corner.

The $Q_{c h u}$ and $Q_{\min }$ value also has been calculated using different equations in [1], [2], [31] and its value listed in Table I. The ring loaded monopole [3] produced the $23.88 \%$ reduction in resonant frequency keeping monopole length unaltered where disc loaded monopole exhibits $34.84 \%$ reduction in resonance frequency in comparison with reference monopole antenna. The ' $k a^{\prime}$ value is 0.49 for miniaturized top hat loaded, complementary split ring resonator based antenna [23]. In current approach the ' $k a$ ' value is 0.48 at 1.29 $\mathrm{GHz}$ with better efficiency. High refractive index meta-material loaded miniaturized monopole [28] produces $16 \%$ bandwidth at frequency $2.50 \mathrm{GHz}$ where as in our case -10 $\mathrm{dB}$ bandwidth is $22.52 \%$. It can be summarized that proposed disc loaded monopole have higher degree of reduction of resonance frequency with high efficiency in comparison with existing literature.

\section{Conclusions}

A novel monopole antenna miniaturization technique is presented based on frequency reduction technique using top loaded dielectric discs; keeping antenna length unaltered for both reference and loaded monopole for better comparison. The monopole is efficiently coupled with one dielectric disc on top of monopole which yields $25.75 \%$ reduced resonant frequency in comparison with a simple monopole. We have introduced one more circular disc on top of monopole to achieve a higher degree of miniaturization. And as a result, proposed antenna achieves $34.84 \%$ reduced resonance frequency with $22.55 \%$ bandwidth and $97.18 \%$ measured efficiency even through the antenna is very simple in configuration without imposing any external matching network. Both the antenna configurations are very efficient and exhibit unperturbed radiation characteristics with respect to unloaded monopole antenna. The whip or monopole antennas are extensively used in cars for their easy integration. The monopole antenna in car can be designed for GPS system, car to car communication, GSM or CDMA operation The Proposed antennas can be used as whip antenna on cars where monopole is cover up with dielectric materials.

\section{Acknowledgement}

The authors would like to thank E and ECE department, IIT Kharagpur for their help and Antenna Research Laboratory, Department of Electronics \& Communication Engineering, Aliah University for Ansys HFSS ver19.2 simulation software. 
Table 1. Antenna performances

\begin{tabular}{cccccccc}
\hline $\begin{array}{c}\text { Antenna } \\
\text { Topology }\end{array}$ & $\begin{array}{c}\text { Resonant } \\
\text { Frequency (GHz) }\end{array}$ & $\begin{array}{c}\text { Miniaturization } \\
\mathbf{( \% )}\end{array}$ & $\begin{array}{c}\text { 10dB bandwidth } \\
\mathbf{( \% )}\end{array}$ & ka & Qchu $_{\text {Qmin }}$ & $\begin{array}{c}\text { Efficiency } \\
\mathbf{( \% )}\end{array}$ \\
\hline Reference Antenna & 1.98 & - & 14.73 & - & - & - & 97.98 \\
Single dielectric disc & 1.47 & 25.75 & 25.75 & 0.55 & 1.65 & 1.60 & 97.41 \\
Double dielectric discs & 1.29 & 34.84 & 34.84 & 0.48 & 2.17 & 2.10 & 97.18 \\
\hline
\end{tabular}

\section{References}

1. McLean, J.S.: 'A re-examination of the fundamental limits on the radiation $\mathrm{Q}$ of electrically small antenna', IEEE Trans. Antennas Propag., 1996, 44, (5), pp. 672-676.

2. Erentok, A., and Ziolkowski, R.W.: 'Metamaterial-inspired efficient electrically small antennas', IEEE Trans. Antennas Propag., 2008, 56, (3), pp. 691-707.

3. Ghosh, B., Haque,SK. M., Mitra, D., Ghosh, S.: 'A loop loading technique for the miniaturization of non-planar and planar antennas,' IEEE Trans. Antennas Propag., 2010, 58,(6), pp. 2116-2121.

4. Hong, W., Sarabandi, K.: 'Low-profile, multi-element, miniaturized monopole antenna', IEEE Trans. Antennas Propag., 2009, $57,(1)$, pp. $72-80$.

5. McVay, J. , Hoorfar, A.: 'Miniaturization of top-loaded monopole antennas using Peano-curves,' in Proc. IEEE Radio and Wireless Symp., 2007 pp. 253-256.

6. Lin, Y. F., Lin, C. H., Hall, P. S. : 'A miniature dielectric loaded monopole antenna for 2.4/5 GHz WLAN applications," IEEE Microw. Wireless Compon. Lett., 2006, 16,(11), pp. 591-593.

7. Noro, T., Kazama, Y.: 'Low profile and wide bandwidth characteristics of top loaded monopole antenna with shorting post', in Proc. IEEE Int. Workshop on Antenna Technol. Small Antennas and Novel Metamater., 2006, pp. 108-111,

8. Haque, SK. M., Parvez, K. M.: 'Slot antenna miniaturization using Slit ,Strip and Loop loading Techniques', IEEE Trans. Antennas Propag., 2017, 65,(5), pp. 2215- 2221.

9. Ghosh, B., Haque, SK. M.; Mitra, D.: 'Miniaturization of slot antennas using slit and strip loading', IEEE Trans. Antennas Propag., 2011, 59, (10), pp. 3922- 3927.

10. Ghosh,B., Haque, SK.M., Yenduri, N.R.: 'Miniaturization of slot antennas using wire loading', IEEE Antennas Wireless Propagat. Lett., 2013, 12, pp. 488-491.

11. Colburn, J. S., Rahmat-Samii, Y.: 'Patch antennas on externally perforated high dielectric constant substrates', IEEE Trans. Antennas Propagat.,1999, 47, pp. 1785-1794.

12. Mongia, R. K., Ittipiboon, A., Cuhaci, M.: 'Low profile dielectric resonator antennas using a very high permittivity material', Electron. Lett., 1994, 30, (17), pp. 1362-1363.

13. Richmond, J. H.: 'Monopole antenna on circular disk', IEEE Trans. Antennas Propagat,1984, 32, (12), pp. 1282 -1287.

14. Simpson, T. L: 'The disk loaded monopole antenna', IEEE Trans. Antennas Propag.,2004,52, (2), pp. 542-550.

15. Hammoud, P. P., Colomel, F.: 'Matching the input impedance of a broadband disc monopole', Electron. Lett., 1993, 29, pp. 406-407.

16. Guo, L.: 'Performances of ultra-wideband disc monopoles in time domain', IET Microw. Antennas Propag., 2007, 1, (4), pp. 955-959. 
17. Friedman, C. H.: 'Wide-band matching of a small disk-loaded monopole', IEEE Trans. Antennas Propag., 1985, 33,(10), pp. $1142-1148$

18. Jung, J.-H., Park, I.: 'Electromagnetically coupled small broadband monopole antenna', IEEE Antennas Wireless Propag. Lett.,2003, 2, pp. 349-351.

19. Lee, J. W., Cho, C. S., Kim, J.: 'A new vertical half disc-loaded ultra-wideband monopole antenna (VHDMA) with a horizontally top-loaded small disc', IEEE Antennas Wireless Propag. Lett., 2005, 4, pp. 198-201.

20. Akhoondzadeh-Asl, L. , Hill, J., Laurin, J.-J., Riel, M.: ‘Novel low profile wideband monopole antenna for avionics applications', IEEE Trans. Antennas Propag., 2013, 61, (11), pp. 5766-5770.

21. Agrawall, N.P., Kumar, G., Ray, K.P.: 'Wide-band planar monopole antennas', IEEE Trans. Antennas Propag., 1998, 46, (2),pp. 294-295.

22. Ansys HFSS ver 19.2, Ansys Corp., Pittsburgh, PA, USA, 2018.

23. NI AWR ver 13, National Instrument Corporation, EI Segundo, CA, USA, 2019.

24. Tang, M-C., Ziolkowski, R. W.,: 'A study of low-profile, broadside radiation, efficient, electrically small atennas based on complementary split ring resonators', IEEE Trans. Antennas Propag, 2013, 61, (1), pp. 4419-4430

25. Oh. J. and Sarabandi. K “Low profile, miniaturized, inductively coupled capacitively loaded monopole antenna," IEEE Trans. Antennas Propag., 2012, 60,(3), pp. 1206-1213, Mar.

26. Pozar, D. M., Kaufman, B., 'Comparison of three methods for the measurement of printed antenna efficiency', IEEE Trans. Antennas Propag., 1998, 36, (1), pp. 136-139.

27. Gudibandi, B.R ,.Murugan, H.A, and Dhamodharan. S.K: 'miniaturization of monopole antenna using High reflective Index metamaterial loading', Int. J. RF Microw. Comput. Aided Eng. 30,(5),5 e22163, 2020.

28. Saraswat, K., Harish, A.R.: 'Split ring loaded monopole antenna', IET Micro. Antennas Propag.,2016, 10, (4), pp. 420-425.

29. C. A. Balanis, Antenna Theory, 3rd ed. New York: Wiley, 2005, pp. 637-641.

30. Mosallaei, H., Sarabandi, K.: 'Magneto-dielectrics in electromagnetics: concept and applications', IEEE Trans. Antennas Propagat., 2004, 52, (6), pp. 1558-1567

31. Sievenpiper, D.F., Dawson, D.C., Jacob, M.M., et al.: 'Experimental validation of performance limits and design guidelines for small antennas', IEEE Trans. Antennas Propag., 2012, 60, (1), pp. 8-19 